\title{
EVALUATION OF THE BALANCE OF OBESE AND NON-OBESE ELDERLY WOMEN BY COMPUTED DYNAMIC POSTUROGRAPHY
}

\author{
E.B. Mantello ${ }^{1}$, M.A. Hyppolito ${ }^{2}$, E. Ferriolli $i^{3}$, N.K. da Costa Lima $a^{4}$ J.C. Moriguti ${ }^{5}$
}

\begin{abstract}
Background: One of the main factors that currently limit the life of the elderly is the imbalance. Computed dynamic posturography, assesses the oscillation of the body by recording the pressure exerted by the feet on the force platform, and allows analyzing the postural reactions secondary to the shift of body weight. Objectives: Evaluate and compare the balance of obese and non-obese elderly women without vestibular symptoms by computed dynamic posturography. Design: prospective trial. Setting: data collected in a university hospital. Participants: 50 elderly females divided into 2 groups according to body mass index (BMI), between 18.5 and $24.9 \mathrm{~kg} / \mathrm{m}^{2}$ for the non-obese group and greater than $30 \mathrm{~kg} / \mathrm{m}^{2}$ for the obese group. Intervention: the participants were submitted to the Synapsys Static \& Dynamic Posturography® test. Measurements: Sensory Organization Test calculates the average proprioceptive, visual and vestibular functions. Data were analyzed statistically by the Fisher exact test. Results: significant differences were observed between the obese and non-obese subjects regarding: maximum amplitude of the anteroposterior displacement of the patient with eyes open and closed, length and surface area used by the patient with eyes open, energy spent with eyes open and closed, proprioceptive activity in the anteroposterior direction, and vestibular activity in the lateral direction. There were a higher percentage of changes in the anteroposterior tests compared to the lateral tests in the obese subjects. Conclusion: The obesity interferes with the body balance in elderly women, especially in situations that require postural control in the AP direction, proprioceptive cues in the AP direction and vestibular cues in the LAT direction.
\end{abstract}

Key words: Postural balance, aged, obesity, acidental falls.

\section{Introduction}

One of the main factors that limit the life of the elderly is imbalance (1).

The evaluation of body balance involves tests that provide information about the ability of an individual to maintain postural stability. One of these tests is computerized dynamic posturography (CDP), which evaluates the oscillation of the body by recording the pressure exerted by the feet on a force platform and permits an analysis of postural reactions secondary to displacement of the center of body mass $(2,3)$.

One of the aspects studied in CDP is the impairment of balance with advancing age. Several authors have studied the balance of healthy elderly people using CDP $(4,5)$. In this respect, most scientific studies indicate that

1, 2. Department of Ophthalmology, Otorhinolaryngology and Head and Neck Surgery, Ribeirão Preto Medical School (FMRP), University of São Paulo (USP), Ribeirão Preto, São Paulo, Brasil; 3,4,5. Department of Internal Medicine, Ribeirão Preto Medical School, University of São Paulo (USP), Ribeirão Preto, São Paulo, Brasil.

Corresponding Author: Erika Barioni Mantello, Department of Ophthalmology, Otorhinolaryngology and Head and Neck Surgery, Ribeirão Preto Medical School (FMRP), University of São Paulo (USP), Ribeirão Preto, São Paulo, Brasil. Av. Bandeirantes, 3600. Campus Universitário. CEP: 14049-900. Phone: 551636022395 Fax: 5516 36022860, Email: erikafga@yahoo.com.br age affects the mechanisms of postural adjustment.

We did not detect references dealing with CDP in obese elderly subjects in the literature. Since research about obesity in the elderly is still insufficient and this is a disease with an unfavorable impact on health and quality of life, and considering the comorbidity usually associated with it and the fact that CDP evaluates quantitatively the main system involved in body balance, the objective of the present study was to assess and compare the balance of obese and non-obese elderly women with no vestibular symptoms using CDP.

\section{Methods}

This was a prospective clinical trial with convenience sampling conducted on 50 volunteer elderly women aged 60 to 89 years, with no otoneurologic symptoms, who were attended at the otorhinolaryngology outpatient clinic of the University Hospital

The study was approved by the Ethics Committee of the University Hospital, protocol $n^{\circ}$. 4915/2008. All patients gave written consent for participation.

The nutritional classification of the subjects was based on the BMI $\left(\mathrm{kg} / \mathrm{m}^{2}\right)$. Regarding the criterion for the nutritional diagnosis for the determination of obesity 
based on the BMI, few specific body weight standards for the elderly are available. In the present study, we opted to characterize the BMI according to the criteria of the WHO (6): low weight $\left(\mathrm{BMI}<18.5 \mathrm{~kg} / \mathrm{m}^{2}\right)$, normal weight (BMI 18.5-24.9 kg/m²), overweight $\left(\right.$ BMI $\left.>25 \mathrm{~kg} / \mathrm{m}^{2}\right)$, and obesity $\left(\right.$ BMI $\left.>30 \mathrm{~kg} / \mathrm{m}^{2}\right)$.

The subjects were divided into two groups according to the following inclusion criteria:

\section{Group 1 ( 25 obese elderly women)}

* Over 60 years of age, with no associated disease (if associated diseases were present, they should be controlled and under specific treatment), giving written informed consente, absence of otoneurological symptoms, BMI of more than $30 \mathrm{~kg} / \mathrm{m}^{2}$.

Group 2 (25 non-obese elderly women)

* Same criteria as for group 1, except a BMI of 18.5 to $29.9 \mathrm{~kg} / \mathrm{m}^{2}$.

Exclusion criteria were:

* Patients with difficulty of intelligibility that would impair the execution of the exam, patients with a BMI of 25 to $29.9 \mathrm{~kg} / \mathrm{m}^{2}$ and lower than $18.5 \mathrm{~kg} / \mathrm{m}^{2}$, Neurological and neoplastic diseases, patients with visual disorders with no appropriate clinical correction (use of corrective lenses on the occasion of the test), Musculoskeletal, orthopedic and psychoemotional changes that would prevent a proper execution of the exam, use of medications acting on the peripheral vestibular system, alcohol intake up to 24 hours before the exam, professionally performed daily physical activity.

All selected patients were then submitted to the CDP exam on a Synapsys Static and Dynamic Posturography ${ }^{\circledR}$ platform (Synapsys, Marseille, France) (7).

Before positioning the patient on the platform, the examiner inserted a new card with the anthropometric data of the patient such as age, gender, weight and height and the results obtained were compared to the normal parameters standardized by the French Association of Posturology (AFP) (7), with each variable being considered individually.

During the test the patients were instructed to remain in the standing position on the force platform, keeping their arms loose along the body and their feet slightly apart and unmoving, in the specific position determined on the platform according to shoes size, and keeping their gaze frontally directed at the horizon. If the patient felt the risk of falling, she was instructed to hold the support bars surrounding the equipment. A second examiner remained behind the patient throughout the test in case she would need support during the exam and in order to prevent accidents. All exams were performed by the same investigator, who gave standardized commands throughout the test.

The goal of the exam in each condition was the maintenance of balance. The patient was instructed to remain unmoving on the platform even when the latter moved. The quantitation of the results obtained ranged from $100 \%$ (no displacement recorded by the sensors of the platform) to $0 \%$, which corresponded to a fall in any direction. Based on the data obtained, the instrument can calculate the mean of each condition and provides an index of proprioceptive, visual and vestibular function.

The exam was based on the following protocol: $1-$ Test of the limit of stability, 2 - Tests of static balance, 3 - Translational tests, 4 - Sinusoidal tests, 5 - Dynamic balance test. After all data (static and dynamic) were obtained, the software calculated the three histograms that represent visual, vestibular and proprioceptive activity and under anteroposterior, posterior and lateral conditions in the Sensory Organization Test (SOT). The normal parameters standardized by the APF for adult and elderly subjects in the tests described here are listed in Synapsys Static \& Dynamic Posturography ${ }^{\circledR}$ Manual version 2.7. 2006. Marseille-France (7).

The characterization of the study population was made by means of descriptive data analysis. To correlate the variables studied, the data were analyzed using this for the Fisher exact test (8). The significance level was set at $\mathrm{p}<0.05$.

\section{Results}

Mean age $(+\mathrm{SD})$ was $69.92 \pm 6.31$ years for the nonobese elderly women, $68.32 \pm 7.48$ years for the obese women, and $69.12 \pm 138.24$ for the total sample. The groups of obese and non-obese elderly women were homogeneous in terms of age $(p=0.59)$. The two groups were also homogeneous regarding the practice of physical activity and the presence of associated diseases ( $\mathrm{p}=0.09$ and $\mathrm{p}=0.74$, respectively).

In view of the wide numerical variability of the results, the data show the absolute and relative frequencies and the Fisher exact test for each parameter evaluated in the two groups.

The data regarding the anteroposterior displacement of the patients with eyes open (AP EO) and with eyes closed (AP EC) were not considered to be statistically significant; however, the AP EO test showed altered results in $60 \%$ of the obese patients and in $32 \%$ of the non-obese patients and the AP EC test showed altered results in $64 \%$ of the obese patients and in $40 \%$ of the non-obese patients.

The data regarding the maximum anteroposterior displacement amplitude of the patients with eyes open (AP AMP EO) and with eyes closed (AP AMP EC) for the obese and non-obese groups were statistically significant in both analyses $(\mathrm{p}<0.01) .84 \%$ of the obese patients and $40 \%$ of non-obese patients showed altered results in the AP AMP EO and AP AMP EC test.

The data regarding lateral displacement of the patients with eyes open (LAT EO) and with eyes closed (LAT EC) for the obese and non-obese groups were not statistically 
significant; however, $36 \%$ of the obese patients and $12 \%$ of non-obese patients showed altered results in the LAT EO test. In the LAT EC test, $20 \%$ of the obese patients and $16 \%$ of the non-obese patients showed altered results.

No statistically significant results were obtained regarding the maximum amplitude of lateral displacement of the patient with eyes open (LAT AMP EO) or closed (LAT AMP EC).

Regarding the LAT AMP EO parameter, the exams were altered in $24 \%$ of the patients in the obese group and in $44 \%$ of the non-obese group. Regarding the LAT AMP EC parameter, the percentage of altered exams was $32 \%$ in both the obese and non-obese groups.

The results of the length parameter used by the patient with eyes open (LSKG EO) and eyes closed (LSKG EC) were not significant for LSKG EO, with altered data observed in $56 \%$ of cases in the non-obese group and in $80 \%$ of cases in the obese group. In contrast, a significant difference was observed between groups regarding LSKG EC $(p<0.01)$, with altered data observed in $44 \%$ of cases in the non-obese group and in $84 \%$ of cases in the obese group.

Regarding the area used by the patient with eyes open (SSKG EO) and eyes closed (SSKG EC), the SSKG EO parameter did not differ significantly, with altered results being obtained in $80 \%$ of the non-obese group and in $92 \%$ of the obese group. In contrast, the results of SSKG EC differed significantly between groups, with altered results being obtained in $44 \%$ of the non-obese group and in $76 \%$ of the obese group ( $\mathrm{p}<0.05)$.

Regarding the energy expended with eyes open (LFS EO) and eyes closed (LFS EC), with both tests showing a significant difference (LFS EO $\mathrm{p}<0.01$; LFS EC $\mathrm{p}<0.05$ ). LFS EO with altered results being obtained in $20 \%$ of the non-obese group and in 64\% of the obese group; LFS EC with altered results being obtained in $24 \%$ of the nonobese group and in $56 \%$ of the obese group.

The percentages of altered exams for the two groups regarding the sensory organization that represent proprioceptive, visual and vestibular activity in the anteroposterior and lateral position. The results of the anteroposterior proprioceptive activity $(\mathrm{p}<0.01)$ and the lateral vestibular activity $(\mathrm{p}<0.05)$ were significant.

The percentages of altered exams for the study groups regarding sensory organization, which represents the proprioceptive activity in the anteroposterior position (altered - obese 68\%, non obese 20\%, p < 0.01) and the proprioceptive activity in the lateral position (altered obese $16 \%$, non obese $16 \%, \mathrm{p}=0.99$ ), visual activity in the anteroposterior position (altered - obese 36\%, non obese $28 \%, \mathrm{p}=0.76)$ and visual activity in the lateral position (altered - obese $24 \%$, non obese $4 \%, \mathrm{p}=0.10$ ), vestibular activity in the anteroposterior position (altered - obese $44 \%$, non obese $36 \%, \mathrm{p}=0.77$ ), and vestibular activity in the lateral position (altered - obese $36 \%$, non obese $8 \%$, p $=0.04$ ).

\section{Discussion}

Studies regarding obesity and its consequences for the population are available in the literature. Some of them have shown the postural and/or osteoarticular changes related to obesity but do not fully clarify the significance of the increase in body mass regarding such changes compared to the non-obese population. Conversely, there is an extensive literature regarding cardiovascular diseases, metabolic changes and respiratory diseases originating from obesity. Thus, the initial hypothesis of the present study was that obese elderly women might show greater difficulties and changes in the results of CDP compared to non-obese elderly women.

Elderly women were selected for the present study because they have a higher prevalence of obesity than men (9).

We did not detect studies in the literature with patient series and methods similar to ours. The studies conducted over the last few years have adopted different instruments and methodologies and no study had been conducted thus far on the obese population. We opted to discuss the results according to the order of the conditions of the evaluations used in CDP Synapsys.

Obesity had a negative influence on the AP AMP EO and EC tests regardless of whether the patients had their eyes open or closed. This permits us also to suggest that obese elderly women need to increase their amplitude of anteroposterior movement in order to maintain their body balance.

Several studies have shown that postural control is reduced with age. As an example, Manfio et al. (10) detected greater excursion of the pressure center in the elderly group than in adult individuals.

Ghulyan et al. (11) conducted a study in order to differentiate groups of healthy young people, healthy elderly people and elderly people with complaints of instability and observed that the only parameter that significantly differentiated the static balance was

AP AMP EO. This parameter also permitted us to differentiate the static balance of the obese and non-obese women in the EO and EC conditions.

The LAT AMP EO test was the only one showing worse results for the non-obese elderly women compared to the obese ones, even though the difference was not statistically significant. The same percentage of altered exams was detected in the LAT AMP EC test. No reports were detected that would permit a discussion of these findings, but we may infer that the increase in body mass did not lead to greater amplitude of the lateral movement in situations of imbalance, as was the case regarding the anteroposterior movement for the obese elderly women.

The results mentioned thus far revealed a predominance of alteration of AP balance compared to LAT balance in both groups, with this being demonstrated more in the group of obese elderly women. Silva et al. (12) observed that young subjects had 
better postural stability than elderly subjects regarding anteroposterior amplitude (12), showing that the AP direction is affected with advancing age, explaining the altered exams in the present population of non-obese elderly women.

Mann et al. (13) reported that the greatest oscillations were detected also in the anteroposterior direction, both for amplitude and for mean displacement of the pressure center. However, in a study of obese children, McGraw et al. (14) detected greater oscillation for the mediolateral balance and concluded that, even though the human body has a greater number of degrees of freedom in the AP direction, children compensated more easily for the imbalance in this direction than in the LAT direction.

It can be seen that the literature is discordant regarding the findings of changes in the indices involved in AP/ LAT balance in young people, adults and healthy and/ or unstable elderly people. It is known that in the anteroposterior direction the first motor response to imbalance occurs by the action of the ankle, followed by the knee and then by the hip. In contrast, in the mediolateral direction, the response occurs only by the action of the hip. In addition, the human body has a larger number of degrees of freedom in the anteroposterior direction compared to the mediolateral direction (14).

The present results show that obese elderly women had significantly greater changes in tests involving AP oscillations compared to LAT oscillations. Since tasks in the AP direction are difficult for non-obese elderly women compared to the LAT direction, the present data indicate that obese elderly women will have even greater difficulties since the excess of adipose mass will impair and slow down the response to oscillation and the stability of the motor response involved.

In addition, obese persons and pregnant women exhibit widening of the support base with separation of the feet when walking, with the feet being displaced sideways, diverging and forming a larger quadrangle that insures new balance positions, causing a waddling and ungainly gait (15). Thus, comparing this statement to our data, we can consider that obese women are better adapted to lateral balance, a fact that justifies lower percentages of alterations in this direction for the obese women and also the greater percentage of altered exams in the non-obese group in the LAT AMP EO/EC test.

Studies on adults have shown that a $20 \%$ increase in body mass reduces the ability to perform rapid adjustments in response to external perturbation in the orthostatic position and increase postural instability (4).

The results of the SSKG EC test showed a statistically significant difference between groups $(p<0.05)$, with the obese women requiring a greater surface to remain stable during the perturbation of body balance.

The LSKG EO/EC + SSKG EO/EC test was altered in 83 cases among the obese women and in 56 cases among the healthy women. When the body is in the erect position it may be compared to a pendulum system that moves through the axis of the ankles. In the presence of overweight, as is the case for obesity, the torque necessary to maintain balance increases, causing increased involvement of the muscles with a motor action in order to bring back the center of the mass to the base of support. This provokes increased values of the variables of balance oscillation (16) and may explain the increase of the surface and length variables for the obese women studied here.

Statistically significant differences were detected between groups in the LFS EO and LFS EC tests, with respective values of $\mathrm{p}<0.01$ and $\mathrm{p}<0.05$. These data permit us infer that obesity impaired the performance of the elderly women in the tests in question, so that these patients must expend a greater quantity of kinetic energy to maintain their body balance.

Regarding the sensory organization for each activity proprioceptive, visual and vestibular - the proprioceptive activity showed statistically significant data $(p<0.01)$, support that obesity reduces the proprioceptive activity in the anteroposterior direction and that elderly women require vestibular and visual sensory information to compensate for the absence of proprioceptive information and thus prevent falls.

Degenerations of the proprioceptive system occur with advancing age, with consequent body instability that explains the changes detected in 20\% of healthy elderly women $(3,17)$. Studies on young and adult obese subjects have demonstrated that the accumulation of adipose tissue can reduce body balance and proprioceptive capacity, contributing to falls (18).

In general, there are two reasons for the proprioceptive alteration of obese persons. The first is that the body, by means of the feet, has mechanoreceptors that receive cutaneous sensations and obesity causes this response to be reduced. In addition, there is an addition of the imbalances, causing greater pressures between the feet and the ground, thus reducing the uptake of sensory information (11).

The present findings agree with those of Pedalini (17) that detected in a study on healthy and symptomatic elderly subjects a preserved proprioceptive system performance compared to the visual and vestibular system. The author stated that the test of sensory integration was not sufficiently sensitive to detect oscillations secondary to the degenerations that occur with aging.

Vestibular activity showed a statistically significant difference $(p<0.05)$ regarding sensory organization for each activity - proprioceptive, visual and vestibular support that the vestibular information in the lateral direction was impaired in obese elderly women, who required the use of visual sensory and proprioceptive information to compensate for the absence of vestibular information in order to prevent falls.

The vestibular information was reduced in the 
obese women in tasks requiring control of balance in the LAT direction. It has been reported that anatomical and physiological changes occur with aging (1) that render the elderly more susceptible to vertigo signs and symptoms, such as reduction of sensory receptors in the semicircular canal, saccule, utricle, and retina, in addition to a reduction of visual and vestibular reflexes.

In view of the above considerations, we may infer that the imbalance of the elderly women studied here was related to obesity. Postural changes do not exclusively occur in obese persons, but affect them more frequently due to the mechanical action of excess body mass and to the increase of the variables that involve balance, especially in the AP condition in relation to the proprioceptive system and in the LAT condition in relation to the vestibular system.

The imbalance and falls of the elderly are largely due to degenerative phenomena peculiar to aging (1). Maintaining one's balance on a surface while rapid displacement movements are required needs a rapid selection of sensory cues and the programming of appropriate postural reactions for body adjustment.

In the present study, CDP was considered to be a rapid test of simple application by the therapist. Some precautions were required for the application of the test to the obese elderly group, especially for women with difficulties in locomotion. However, these deficits did not prevent the application of the test since parameters such as frequency and velocity can be changed manually.

We suggest a program of balance rehabilitation for the population of obese elderly women under the guidance of an interdisciplinary team that would provide medical, nutritional, speech therapy, physiotherapy and physical education follow-up. It would also be interesting to conduct a longitudinal study of obese elderly women with CDP evaluation in a first phase and after the implantation of a program of weight loss and rehabilitation.

\section{Conclusion}

Our study support that the obesity interferes with the body balance in elderly women, especially in situations that require postural control in the AP direction, proprioceptive cues in the AP direction and vestibular cues in the LAT direction.

Ethical Standards: The experiments described in this manuscript comply with the current laws of Brazil.

Acknowledgments: - Fundação de Apoio ao Ensino, Pesquisa e Assistência (FAEPA) of Ribeirão Preto Medical School (FMRP), University of São Paulo (USP), Ribeirão Preto, São Paulo, Brasil. - We also acknowledge the substantial scientific contribution made by women participating in this study.

Conflicts of interest: The authors attest that there is no conflict of interest (financial and/or personal) that could affect the proper way to work this manuscript.

\section{References}

1. Ruwer LS, Rossi AG, Simon LF. Balance in the elderly. Rev Bras Otorrinolaringol 2005; 71: 298-303.

2. Black FO. What can posturography tell us about vestibular function, Ann N Y Sci 2001; 940:446-64.

3. Novalo ES, Pedalini MEB, Bittar RSM, Lorenzi MC, Bottino MA. Computerized Dynamic Posturography: Quantitative Evaluation in Patients with Vestibulopathy Treated with Vestibular Rehabilitation. Int. Arch. Otorhinolaryngol 2008; 12:253-257

4. Borah D, Wadhwa S, Singh U, Yadav SL, Bhattacharjee M, Sindhu V. Age related changes in postural stability, Indian J Physiol Pharmacol. 2007; 51: 395-404.

5. Cohen H, Heaton L, Congdon SL, Jenkins HA. Changes in sensory organization test scores with age. Age Ageing 1996; 25:39-44.

6. WHO - World Health Organization. Obesity: preventing and managing the global epidemic. 1998. Geneva: WHO.

7. Synapsys Static \& Dynamic Posturography® Manual version 2.7. 2006. Marseille-France.

8. SAS/STAT® User's Guide, Version 9 (2002-2003) Cary, NC: SAS Institute Inc.

9. Marques APO, Arruda IK, Espírito SantoAC, Raposo MC, Guerra MD, Sales TF. Prevalence of obesity and associated factors in older women. Arq Bras Endocrinol Metab. 2005; 49:441-448.

10. Manfio EF, Muniz MA, Rabello BV. Relationship between static balance and ground reaction force. 2005. 11th Brazilian Congress of Biomechanics, João Pessoa.

11. Ghulyan V, Paolino M, Lopez C, Dumitrescu M, Lacour M. A new translational platform for evaluating aging or pathology-related postural disorders. Acta Oto-Laryngol 2005; 125: 607-617.

12. Silva JB, Sousa PN, Lima ES, Teixeira LA. Comparison of postural control in adults and elderly subjects: task dependency and visual information. Aging and Physical Education Perspectives and Challenges. 2002. São Paulo, pp.175-78.

13. Mann L, Teixeira CS, Lopes LFD, Mota CB. Evaluation of the force center during static balance in academic physical education. 5th Exhibition of Scientific Initiation, Postgraduate and Research at the University of Caxias do Sul. 2005. Vacaria, RS.

14. Mc Graw B, Clenaghan BA, Williams HG, Dickerson J,. Ward DS. Gait and postural stability in obese and nonobese prepubertal boys. Arch Phys Med Rehabil 2000; 81: 484-9.

15. Alvarenga GM, Gambá HR, Moser AD. Kinematic analysis pre and postoperative patients undergoing bariatric surgery: a preliminary study. Proceedings of the 21st Brazilian Congress of Biomedical Engineering. 2008. Salvador, BA. pp. 225-8.

16. Hue O, Simoneau M, Marcotte J, Berrigan F, Doré J, Marceau P et al. Body weight is a strong predictor of postural stability. Gait Posture. 2007. 26: 32-8.

17. Pedalini MEB. Assessment of older people with and without vestibular symptoms by computerized dynamic posturography. 2005. Thesis. Faculty of Medicine, University of São Paulo. 